\title{
Quand la céramique de Limoges vient au secours des océans
}

\section{When Limoges ceramics comes to the rescue of the oceans}

\author{
Guillaume Lévêque ${ }^{1}$, David Branthome ${ }^{2}$ \\ ${ }^{1}$ responsable céramique, société I.CERAM, ceramiques@iceram.fr \\ ${ }^{2}$ directeur de l'Aquarium de Limoges, david.aquariumlimoges@gmail.com
}

\begin{abstract}
RÉSUMÉ. La protection et le développement des récifs coralliens est un enjeu écologique majeure actuel. En effet, la dégradation importante de ces derniers, due essentiellement au réchauffement climatique et à la pollution humaine, nuit à la biodiversité. Par conséquent, l'aquarium de Limoges et le laboratoire I.Ceram, fabricant de substituts osseux en céramique inerte réalisent des travaux de recherche portants sur l'utilisation de l'alumine poreuse comme support de bouturage pour les coraux. Les travaux réalisés ont montré que ce type de support est totalement adapté à la repousse du corail et permet un développement vivant au cœur de la céramique pour former une " pierre vivante ».

ABSTRACT. The protection and the development of coral reefs is a major ecological issue today. Indeed, the significant degradation of these reefs, mainly due to global warming and human pollution, is damaging biodiversity.

Consequently, the Limoges Aquarium and I.Ceram laboratory, a manufacturer of inert ceramic bone substitutes, are carrying out research into the use of porous alumina as a cutting support for corals.

The work carried out has shown that this type of support is totally adapted to the regrowth of coral and allows a living development in the heart of the ceramic to form a "living stone".

MOTS-CLÉS. Biodiversité, corail, bouturage, céramique, substitut osseux.

KEYWORDS. Biodiversity, coral, cuttings, ceramic, bone substitute.
\end{abstract}

\section{Introduction}

Les récifs coralliens ne représentent que $0,16 \%$ de la surface de nos océans $\left(280000 \mathrm{~km}^{2}\right)$ mais hébergent plus de $30 \%$ de la biodiversité marine (plus de 1 million d'espèces animales et végétales hébergées par les récifs coralliens).

La France est le seul pays à avoir des récifs coralliens dans les 3 océans. En effet, 10\% des récifs coralliens sont français.

Le corail qui ressemble à un végétal ou un minéral est un animal dur, mou ou gélatineux nommé polype qui vit fixé sur un support. Le polype est petit (de $1 \mathrm{~mm}$ à $10 \mathrm{~cm}$ ) et possède des tentacules, une bouche, un anus, et un gros estomac. Il vit plutôt la nuit où il se déploie, il balance ses tentacules dans le courant et capture ainsi sa nourriture. Le jour, il se rétracte dans son calice, il absorbe le calcaire dissous dans l'eau de mer pour fabriquer son squelette.

Il vit en colonie et se fixe au fond de l'eau. Le récif corallien, constitué de milliers de polypes, protège la côte de la houle. Chaque polype contient une algue photosynthétique, la zooxanthelle, qui a le même rôle que les arbres. C'est pourquoi, à eux seuls, les récifs coralliens produisent plus d'O2 que la forêt amazonienne.

Lorsque la mer se réchauffe trop, le corail rejette l'algue, blanchit, et n'est plus nourri, donc il meurt : c'est ce que l'on nomme le «blanchissement des coraux ».

Le corail est très fragile et peut être dégradé par des causes naturelles tels que les cyclones, la houle, les maladies mais aussi des causes où l'homme est responsable tels que la pollution de l'eau, les mauvais traitements des eaux usées, les mauvaises techniques de pêche, les bateaux jetant l'ancre sur des fonds coralliens. Par conséquent, 90\% du corail est aujourd'hui dégradé. 
De plus, le corail pousse lentement, de 1 à $20 \mathrm{~mm}$ par an.

D'un point de vue économique les coraux fournissent des ressources et services estimés à 375 milliards de dollars chaque année et soutiennent plus de 850 millions de personnes dans le monde par la nourriture et l'emploi.

En effet, $1 \mathrm{~km}^{2}$ de récif contient de 5 à 15 tonnes de poissons et crustacés Que ce soit pour préserver la biodiversité marine ou également pour préserver nos côtes(les coraux protègent $150000 \mathrm{~km}$ de côtes dans 100 pays) et nos climats, il est primordial de protéger les coraux.

\section{Hypothèses}

L'aquarium de Limoges ainsi que d'autres aquariums européens travaillent depuis plusieurs années sur la reproduction des coraux par bouturage pour peupler leurs bassins sans faire de prélèvement dans la nature mais également pour mieux les comprendre et donc les protéger.

Le bouturage se fait habituellement sur un support en PVC ou en ciment. Cette méthode peut être optimisée car le corail, en grandissant, englobe son support qui fera partie intégrante de l'animal. Lorsque ce corail bouturé se fait manger par un poisson, ce dernier ingère du PVC et du ciment...

Qui nous dit qu'un jour il ne se retrouvera pas dans nos assiettes?

Une collaboration avec la société I.Ceram, basée à Limoges, a débuté en fin d'année 2018. La société I.Ceram est spécialisée dans la conception, la fabrication et la commercialisation de substituts osseux en céramique poreuse déjà implantés à plus de 7000 patients à travers le monde depuis sa création. Cette céramique poreuse (alumine), inerte chimiquement et non dégradable sera donc totalement neutre pour l'environnement.
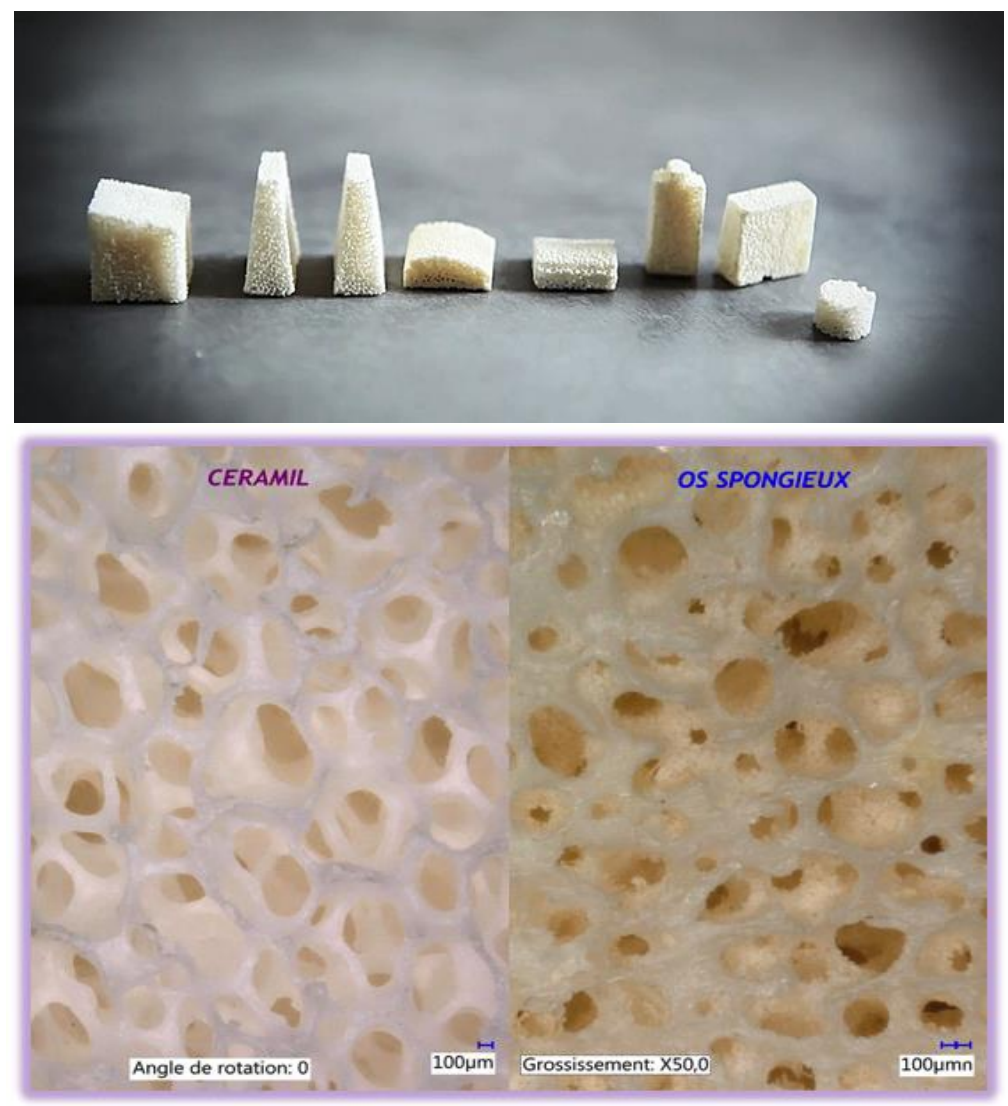

Imaginons un jour que la céramique de Limoges permettent de réparer des brèches dans la grande barrière de corail et permettent aux coraux de s'y fixer de façon pérenne? 


\section{Essais réalisés}

Les résultats sont au delà de nos espérances.

Actuellement, les coraux sont fixés soit avec du fil nylon soit avec des colles synthétiques. Des tests ont été réalisés pour fixer les coraux sur l'alumine soit par impaction soit à l'aide de fils chirurgicaux résorbables pour avoir un impact entièrement neutre pour l'écosystème aquatique. Les résultats ont montré une fixation primaire de qualité et le corail repousse autour du fil de suture.

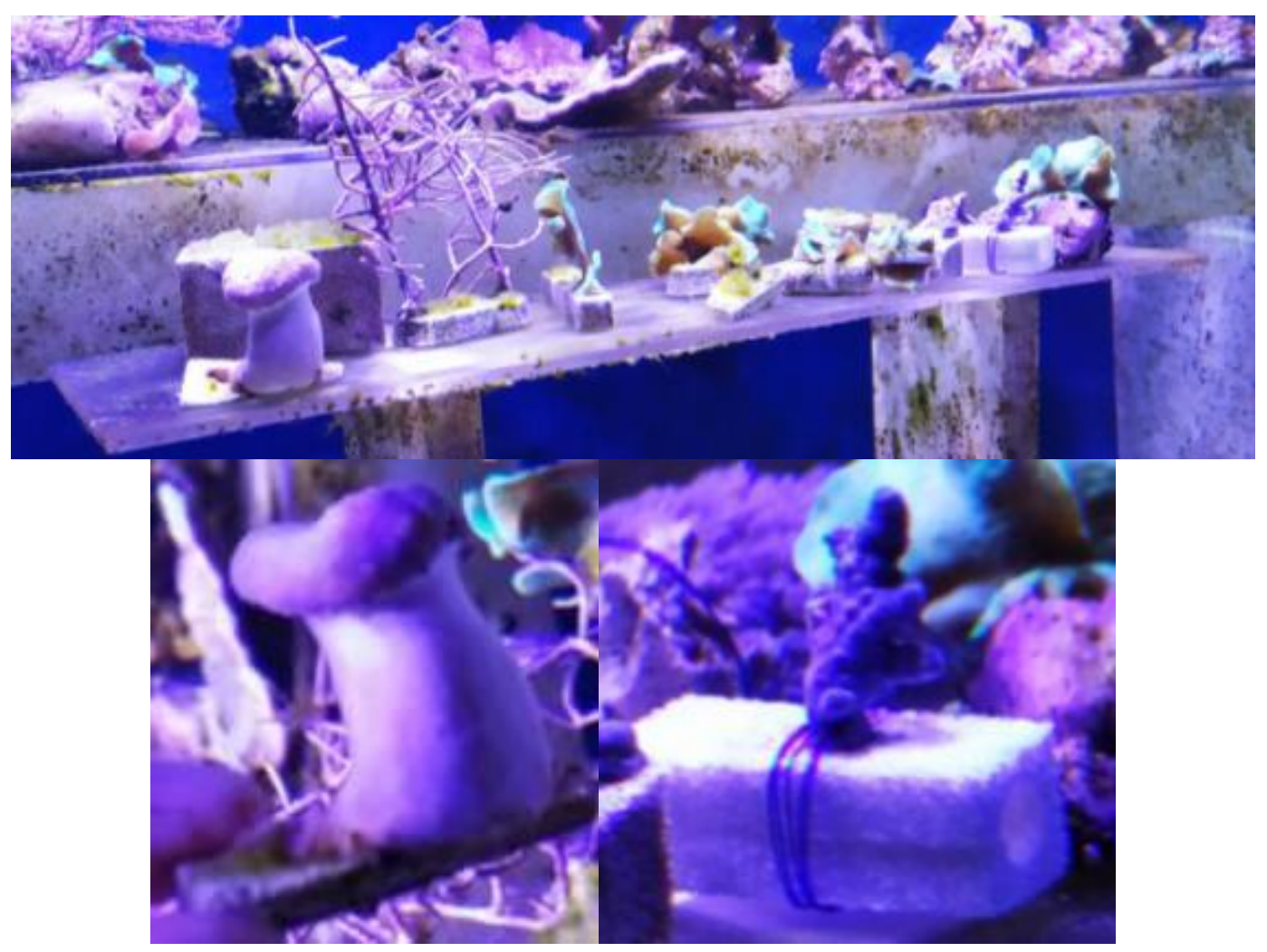

Le support céramique est adapté à la fixation des coraux qu'ils soient durs ou mous et les coraux semblent se fixer plus durablement sur la céramique. La fixation en surface sur 1 à $2 \mathrm{~mm}$ de profondeur est suffisante pour un bon maintien mécanique. En effet, leur ancrage dans la porosité de la céramique est de qualité.

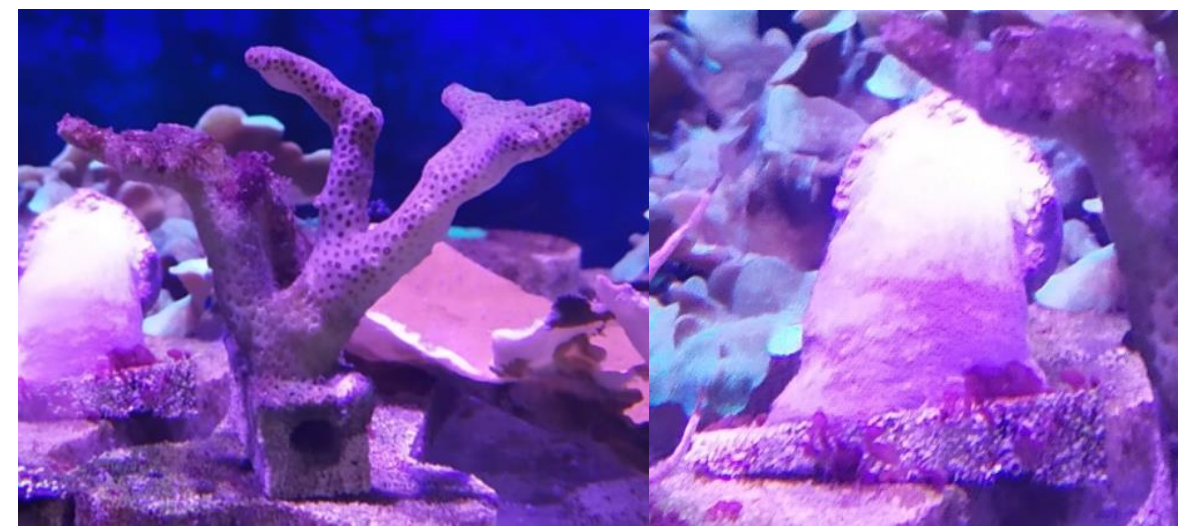

Non seulement la fixation des coraux se déroulent parfaitement bien mais le support héberge également une multitude de vie microscopique grâce à sa porosité. Les algues supérieures se fixent sur le support céramique et entrent dans la chaîne alimentaire. 


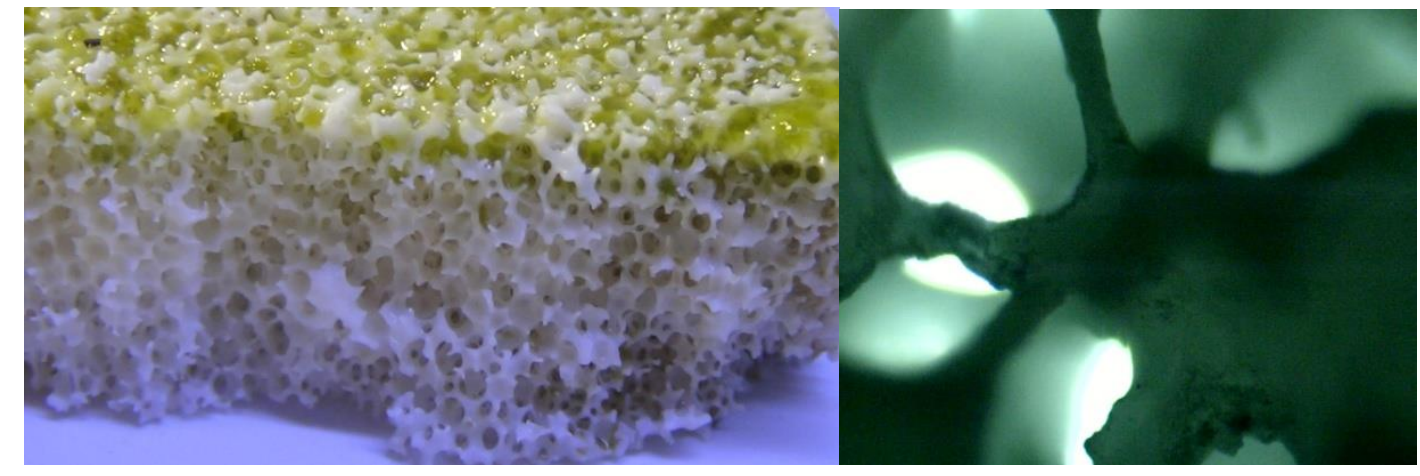

La vie se développe au cœur de la céramique pour former une «pierre vivante ».
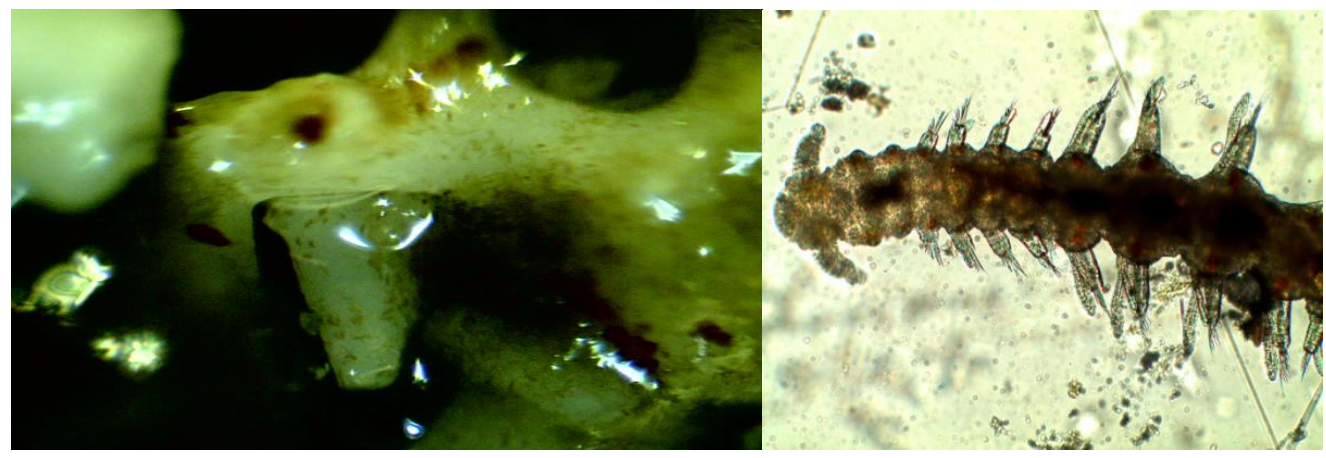

La solution du bouturage des coraux présente beaucoup d'avantages mais aussi quelques inconvénients comme l'appauvrissement de la génétique car le bouturage est un clonage, un risque sur la biodiversité par le choix des espèces, un risque de mortalité importante si maladie, parasite ou aléas climatiques. Il est donc indispensable de travailler en parallèle sur la reproduction sexuée des coraux et à la fixation des larves pour faire un brassage génétique indispensable à la survie à long termes des récifs coralliens et à la biodiversité marine.

Cette première phase d'étude concluante sera poursuivie par des analyses de quantité, qualité et vitesse de repousse du corail en fonction du matériau support. De nouvelles géométries de supports seront aussi développées afin de favoriser le bouturage des coraux. Un bassin spécialement dédié à cette étude sera intégré au sein de l'aquarium de Limoges.

Tous ces travaux s'inscrivent également dans le grand projet de l'Aquarium de Limoges. En effet, une étude a débuté pour déménager l'aquarium vers une structure plus grande mais surtout tourner vers le développement durable où le visiteur sera immergé dans le monde aquatique de façon totalement innovante et ainsi comprendra l'intérêt de protéger notre planète.

La céramique sera au cœur de ce projet non seulement pour les décors mais aussi pour le système de filtration et de construction de ce bâtiment. 